\title{
Percutaneous radiofrequency and microwave ablation in the treatment of renal tumors - 10 years of experience
}

\author{
Petr Dvorak ${ }^{1}$, Petr Hoffmann ${ }^{1}$, Milos Brodak², Josef Kosina², Jaroslav Pacovsky², Jan Raupach ${ }^{1}$, Antonin Krajina ${ }^{1}$ \\ ${ }^{1}$ Department of Radiology, University Hospital, Hradec Kralove, Czech Republic \\ ${ }^{2}$ Department of Urology, University Hospital, Hradec Kralove, Czech Republic
}

Videosurgery Miniinv 2017; 12 (4): 394-402

DOI: https://doi.org/10.5114/wiitm.2017.72322

\begin{abstract}
Introduction: The standard radical treatment of renal cell carcinoma is surgical resection, but it is not suitable for patients with serious medical comorbidities and solitary kidney tumors. Minimally invasive ablation techniques could be an appropriate therapeutic alternative.

Aim: To retrospectively evaluate the technical success, mid-term and long-term efficacy and safety of radiofrequency and microwave ablation in patients with small renal tumors.

Material and methods: Over the course of 10 years, 91 ablation procedures in 64 patients for 68 tumors, of size 12$60 \mathrm{~mm}$, were performed using only conscious sedation. These ablations were done under the guidance of computed tomography. We treated 41 males and 23 females with solitary kidney tumors (14 cases) and tumors in non-surgical candidates (54 cases).

Results: In 50 (73.5\%) tumors single treatment was successful; in 13 (19.1\%) cases a second procedure was used successfully, and in the 5 largest tumors (sizes $45-60 \mathrm{~mm}, 7.4 \%$ ) a third treatment was necessary. Within the follow-up 10 (15.6\%) patients died, but none due to metastatic renal cell carcinoma. Only 1 serious complication was observed - retroperitoneal and psoatic hematoma. Early recurrence occurred in 18 (26.5\%) tumors. Late recurrence was detected in 5 (7.4\%) cases. In all cases complete local control of the renal tumors was reached.

Conclusions: Percutaneous ablation is a very effective treatment for patients with small renal tumors of the T1a group with a minimal complication rate.
\end{abstract}

Key words: radiofrequency ablation, microwave ablation, small renal tumors, minimally invasive therapy.

\section{Introduction}

Renal tumors constitute $2-3 \%$ of all neoplasms. The most important type is renal cell carcinoma (RCC), which represents up to $95 \%$ of all renal malignancies. The incidence of this tumor has continued to increase within the last decades. About 35,000 new cases were diagnosed in the USA in the early 2000s. Furthermore, in 2009 there were approximately 57,760 new cases and 62,700 new cases in 2016. The American Cancer Society's most recent estimates for 2017 are about 63,990 new cases with about 14,400 deaths from this disease [1-3]. In the Czech Republic the most recent incidence figures were about 27 new cases per 100,000 people, or about 2,800 new renal malignancies in 2016. Renal cancer is a disease that occurs frequently and is accompanied with high morbidity and mortality rates. Renal cell carcinoma is diagnosed in elderly people, between 50 and 70 years of age; men are two or three times more likely to be affected [4]. Despite the fact that small incidental tumors are detected, there is still a high incidence of advanced tumors being diagnosed, a consequence of the relatively 
long asymptomatic progress of this disease. The diagnostic possibilities are more accurate and more readily available, and the treatment makes doubtless progress, but the mortality rate remains almost stationary.

\section{Aim}

The aim of the study was to retrospectively evaluate the technical success, mid-term and long-term efficacy and safety of radiofrequency and microwave ablation in patients with small renal tumors.

\section{Material and methods}

Within the last 10 years, from February 2007 to February 2017, a group of 64 patients was prospectively observed. This group included 41 (64\%) men and 23 (36\%) women, aged 52 to 85 years (median: 76 years of age). Percutaneous ablations were performed for 68 tumors of size $12-60 \mathrm{~mm}$ in diameter (median length: $27 \mathrm{~mm}$ ). All of these interventional procedures were performed under computed tomography (CT) guidance, except for 5 cases which were performed with CT fluoroscopy. Emphasis was put on the correct indication establishing patients' preparation, technical aspects of the whole procedure, possible complications, and their prevention and monitoring of the success rate. The start of the study for each patient began with the first ablation; patient monitoring ended at end of the study (February 2017), the last known patients' control with imaging method or the day of death. The observation lasted from 4 to 92 months (median length 51 months).

The indicated cases were divided into two groups - patients with tumors of a solitary kidney (14 tumors, $20.6 \%$ ) and tumors of non-surgical candidates due to many medical comorbidities and high risk of general anesthesia (54 tumors, 79.4\%). According to renal localization we treated exophytic tumors in 38 (55.9\%) cases and intraparenchymal tumors in $30(44.1 \%)$ cases. Centrally localized neoplasms were not indicated due to high risk of serious postprocedural complications.

The diagnoses were established on the basis of contrast medium enhanced imaging methods, CT in 58 (85.3\%) tumors and magnetic resonance (MR) in 8 (11.8\%) neoplasms. We used the CT scanner Siemens Somatom Definition AS Plus (Siemens, Forchheim, Germany) and MR Siemens Magnetom
Avanto 1.5 Tesla (Siemens, Forchheim, Germany). A mass was diagnosed as a tumor that consisted of a contained character, hypervascularized in both the arterial and nephrographic phase of more than 50 Hounsfield units (HU). In 2 (2.9\%) cases the diagnosis could not be established by imaging methods; thus we performed a percutaneous biopsy and the renal cell carcinoma was verified. All these parameters are also adequate for surgical treatment. All the patients were indicated on the basis of the multidisciplinary council (radiologist, urologist, oncologist, and anesthesiologist).

Fully informed consent was obtained in all cases with an explanation of the procedure, the disease, possible complications, and their possible solutions. The procedures were performed using StarBurst instruments (AngioDynamics, Queensbury, New York, USA) of radiofrequency ablation (61 cases, $89.7 \%$ ) or the Acculis system (AngioDynamics, Queensbury, New York, USA) of microwave ablation (7 cases, 10.3\%). Targeted tissue temperature was set and controlled between $100^{\circ} \mathrm{C}$ and $110^{\circ} \mathrm{C}$, where the duration of ablation varied between 5 and $12 \mathrm{~min}$. All procedures were performed under the guidance of CT.

All procedures were made using only local anesthesia (trimecaine, Zentiva, Prague, Czech Republic) and conscious sedation using 1\% propofol (Fresenius Kabi AB, Bad Homburg, Germany). Parameters of blood coagulation, international normalized ratio (INR, lower than 1.5) and activated partial thromboplastin time (aPTT, lower than 1.3) were noted before the treatment by an anesthesiologist. After the intervention we performed track ablation, the thermal destruction of the ablation needle track, in order to prevent any tumorous cells seeding into retroperitoneum and soft tissues of the lumbar region, stopping any small bleeding. Then the point of insertion into the skin was cleaned with surgical scrub and a series of CT scans were performed to exclude the possibility of early complications. The duration of the whole procedure including all preparations never exceeded $60 \mathrm{~min}$. The patients were monitored for the next $3 \mathrm{~h}$ by the department of anesthesiology, then by the standard department of urology. The next morning they were discharged from the hospital after clinical, laboratory, and ultrasound examinations.

In two technically difficult cases due to the narrow relationship between the renal tumor and the large bowel, hydrodissection was performed. To in- 
crease the safety of the procedure, an instillation of about $150 \mathrm{~mm}$ of saline was made into the surrounding area of the tumor using a Chiba needle before the ablation. The goal of this step was to artificially expand the initially narrow peritumoral space to prevent a thermal colonic injury (Photos $1 \mathrm{~A}-\mathrm{F})$. In cases of the two largest tumors we used a combination approach - percutaneous ablation $24 \mathrm{~h}$ after the selective arterial embolisation using N-butyl-2-cyanoacrylate (Histoacryl, B. Braun Medical, Melsungen, Germany) and Lipiodol (Lipiodol Ultra-fluide, Guerbet, Roissy, France) in a ratio of 8 to 1 . These techniques were used in the year 2008, a time when microwave ablation was not yet available.

After treatment care was provided in cooperation with the urologist at elected intervals with control of clinical status, laboratory parameters (serum level of creatinine, especially in the group with solitary kidney tumors) and imaging methods (contrast medium enhanced $\mathrm{CT}$ in 56 patients and MR in 8 patients). Imaging methods were crucial for establishing the success rate. They were performed at intervals of 1 , 6 , and 12 months after the initial procedure and then once each year thereafter. Computed tomography examination was preferred in the patients with normal renal functions (normal serum level of creatinine). Other patients were recommended for MR imaging. It was considered a success with the absence of opacification during the ablation after an intravenous contrast medium was administered on CT, MR or scarlike retraction of the surrounding renal parenchyma.

If there was a suspicion of non-ablation or a residual tumorous process, we immediately performed the next ablation procedure. This technique was not different from the initial intervention; it only focused on the localization of residual tumorous tissue.

\section{Statistical analysis}

For the basic quantitative statistical evaluation, the median and interval data were used. These parameters were correlated with complications and recurrence using non-parametric tests of Mann-Whitney and Kolmogorov-Smirnov. Qualitative statistical data were descriptively evaluated and in relationship with quantitative parameters using the $\chi^{2}$ test. For survival rate evaluation, the Kaplan-Meier empirical curve was used and was correlated with complications and recurrence. Statistical significance was established at the level $p=0.05$ (Figure 1). The sta- tistical program NCSS 9 (NCSS, LLC, East Kaysville, Utah, USA) was used.

\section{Results}

The duration of observation and controlling of all patients varied from 4 to 92 months (median length: 51 months, average lengt: 46.7 months). Within this period we performed 91 percutaneous ablation sessions for 68 tumors in 64 patients under the control of computed tomography guidance.

In 50 (73.5\%) tumors single ablation was successful, in 13 (19.1\%) cases a second procedure was also performed, and in the 5 largest tumors (length from 45 to $60 \mathrm{~mm}, 7.4 \%$ ) a third treatment was needed. Tumors smaller than $27 \mathrm{~mm}$ in diameter always had single treatment success (Photos $2 \mathrm{~A}-\mathrm{F}$ ).

Within the treatment care observation, 10 (15.6\%) patients died. In 2 cases it was due to nonsmall cell lung carcinoma (NSCLC), in 2 cases due to colorectal cancer, and in 1 case each of prostate cancer, cholangiocarcinoma, hepatic failure, acute myocardial infarction, congestive heart failure, and exacerbation of chronic obstructive pulmonary disease. None died due to metastatic renal cell carcinoma. All of these cases were verified by an autopsy.

The serum level of creatine in the renal functions was monitored in the group of solitary kidney tumors. The pre-procedural levels never exceeded 15\% at 6 months after the initial treatment.

Early complications consisting of peritumoral hematoma only occurred in 6 cases out of 91 interventions up to $24 \mathrm{~h}$ after the ablation (6.6\%). A conservative course of treatment was suitable in all cases. All these hematomas were resolved within the follow-up regular treatment care observation; no surgical intervention was needed (Photos $3 \mathrm{~A}-\mathrm{C}$ ). Additional complications such as abscesses, seromas, urinomas, collecting duct strictures or peritoneal tumorous spreading were never detected.

Early recurrence, i.e. the presence of tumorous tissue up to 3 months after initial treatment, was verified in 18 (26.5\%) cases. The amount of residual tumorous tissue depended only on the size parameters. Re-ablation was performed in 13 cases within the period after the first follow-up (Photos 4 A-F). In cases of the 5 largest tumors an additional procedure was necessary.

Late recurrence, i.e. the presence of residual tumorous tissue more than 36 months after the 

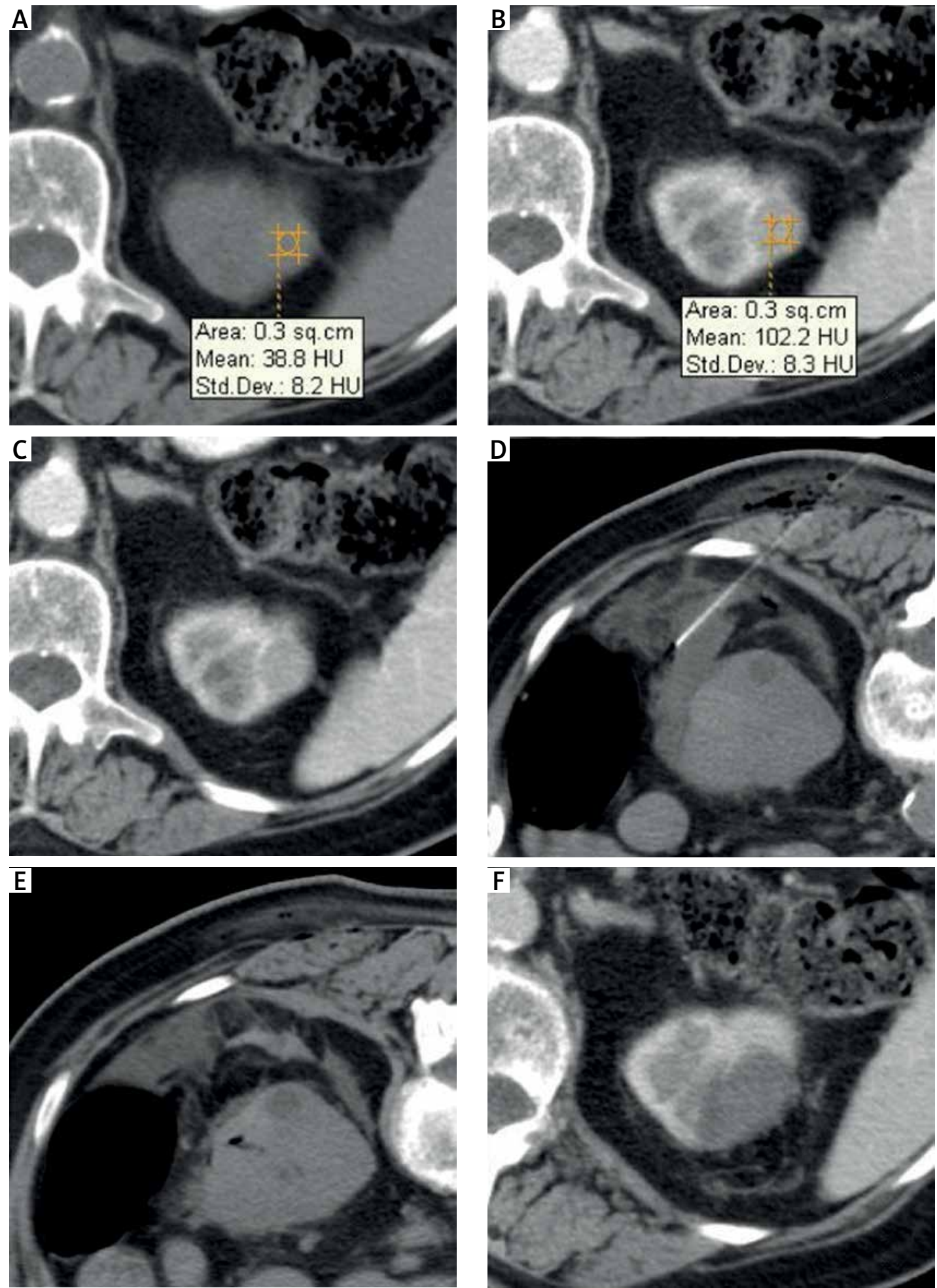

Photo 1. Hydrodissection. Small hypervascularized tumor in upper pole of the left kidney on nonenhanced CT (A) and after contrast medium intravenous administration (B) and adjacent localization of large bowel (C). The instillation of $150 \mathrm{~mm}$ of saline using Chiba needle (D), patient in prone position. Control CT scan right after procedure reveals none complication (E). After ablation treatment care in interval 1 month verifies none residual tumorous tissue $(F)$ 


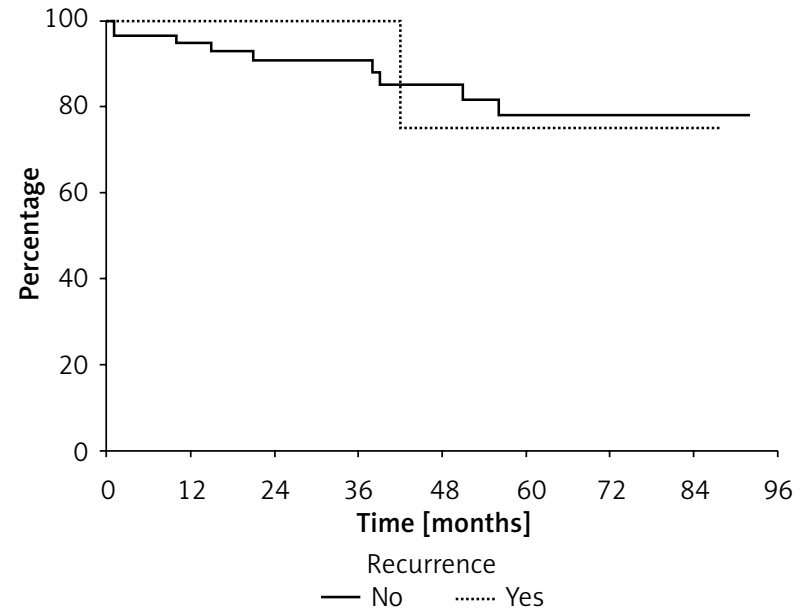

Figure 1. Kaplan-Meier empirical curve of survival rate correlated with recurrence (dotted line) and without recurrence (continuous line). The statistically significant difference was not established ( $p=0.9139)$

initial treatment, was confirmed in 5 (7.4\%) cases. Two cases of recurrence were treated with percutaneous radiofrequency ablation. Two residual tumors were treated with nephrectomy, and in 1 case surgeons successfully completed nephron-sparing intervention. Due to the small amount of residual tumorous process left after ablations and after treatment care, the recurrence does not reduce patients' survival.

\section{Discussion}

The standard radical treatment of renal cell carcinoma is total nephrectomy. The most preferred approaches in the past preserved renal functions. However, new surgical techniques have evolved under the umbrella term nephron-sparing surgery (NSS) into such practices as laparoscopy, robotic resection, and many other various modified approaches to the affected kidneys [5]. Many of these patients have serious medical comorbidities and general anesthesia is contraindicated. With this reasoning, non-surgical methods were also developed. These techniques include destructive tissue methods, and include modality groups such as radiofrequency ablation (RFA), microwave ablation (MWA), cryoablation, laser-induced thermal therapy (LITT), high-intensity focused ultrasound (HIFU), and irreversible electroporation (IRE) [6-10]. As renal cell carcinoma is chemoresistant and radioresistant, these types of treatment were not suitable.
There are two major directions when establishing a diagnosis: with or without a pre-procedural biopsy. A biopsy is relatively risky due to the possibility of serious bleeding from the hypervascularized tumor. A positive histological result would reveal a tumor up to $40 \mathrm{~mm}$ in length, an unsuitable size for ablation, which requires small tumors to be successful [11]. According to many studies in the literature, it is a long-established fact that about $10 \%$ of resected kidney tumors are histologically benign types of oncocytoma or lipid-poor angiomyolipomas [12]. We used the same diagnostic schema, which is adequate for surgical intervention, imaging-based diagnosis, and a biopsy, which is reserved for particularly ambiguous cases. In the future there is the question about double-canal instrument construction, which entails using two ducts, the first duct for the biopsy and the second one for the ablation.

Tumors occurring in central renal parts are not suitable for ablation due to the high risk of hemorrhage or collecting duct injury. Intraparenchymal and exophytic tumors are appropriately located for ablation. The close relationship of the renal tumor and any other intra-abdominal structure can cause thermal injury to the surrounding non-tumorous tissue. The tumor can be separated from the organs by instilling a medium between them. Saline or glucose solutions are often used. If a fluid is used, the procedure is called hydrodissection. $\mathrm{CO}_{2}$, hyaluronic acid gel or a balloon catheter can also be used to increase the safety of the intervention [13].

The success of the ablation procedure in the vast majority of cases depends on the tumor size. The treatment of neoplasms up to $30 \mathrm{~mm}$ in diameter has a nearly $100 \%$ success rate with a single procedure $[6,7]$. There is often residual presence of larger tumors after ablation. If there is a small amount of residual tumorous tissue, the next ablation procedure is prepared for complete tissue destruction. Tumors larger than $50 \mathrm{~mm}$ are not suitable for ablation. The other tumor parameters, such as histological character, vascularization type, or coexisting chronic renal disease, are not associated with ablation procedure success.

Nephron-sparing surgery has an advantage because of the possibility of histological verification and evaluation of surgical margins. If these margins are negative for a tumorous process, then the treatment is considered radical [14]. The disadvantages 

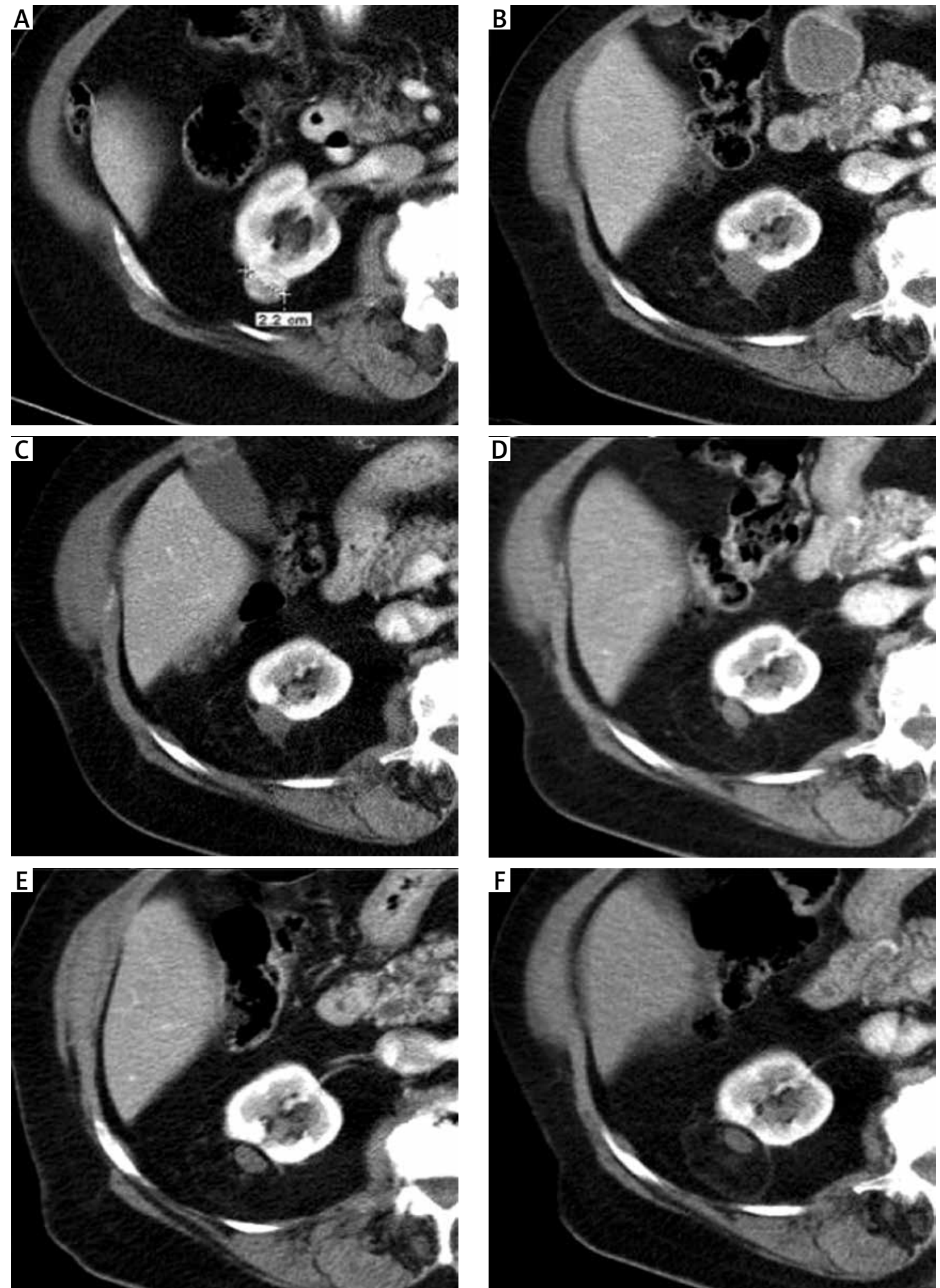

Photo 2. Successful ablation of small renal tumor. Computed tomography examination with intravenous administration of contrast medium. Preprocedural finding of the hypervascularized tumor of length $22 \mathrm{~mm}$ (A). After ablation treatment care in interval 1 month (B), 6 months (C), 12 months (D), 24 months (E) and 36 months (F). The absence of opacifying and scar-like retraction of surrounding renal parenchyma are revealed 

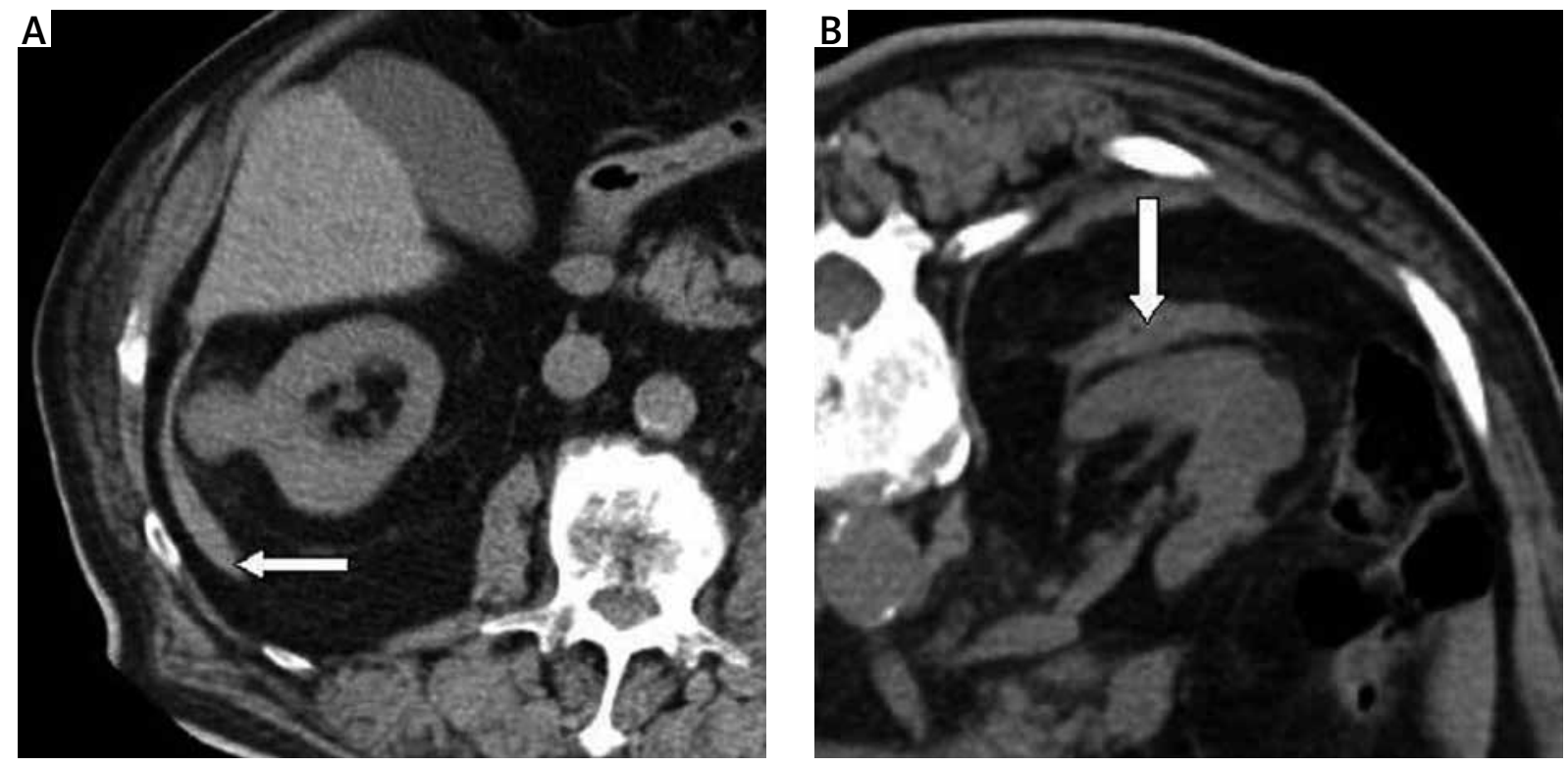

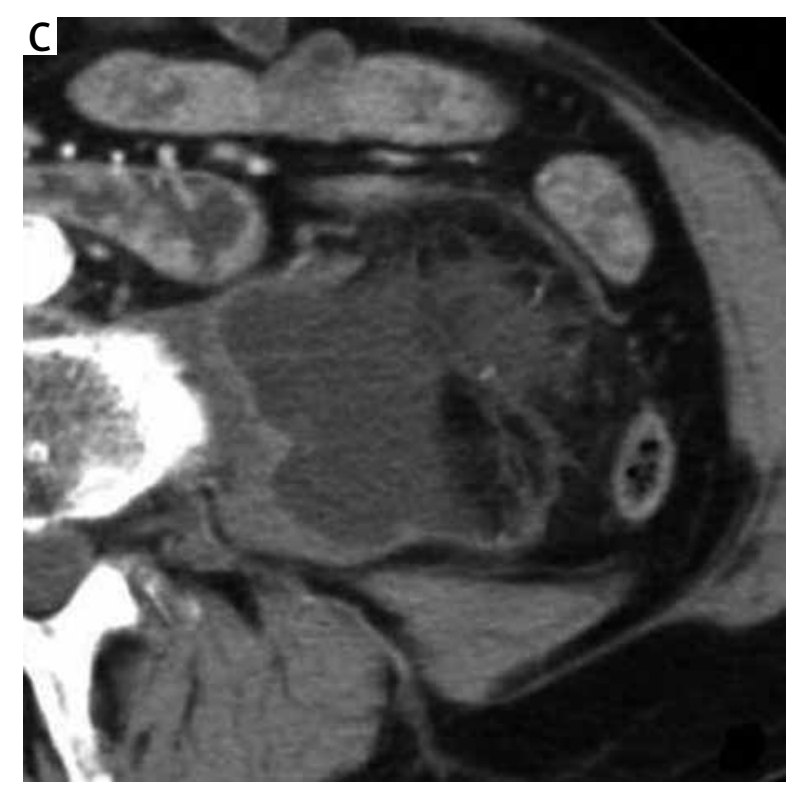

of surgical treatment include an operation wound in the lumbar region, which is often painful and requires general anesthesia and may lead to further complications such as seroma, urinoma, inflammation, or vascular impairment (pseudoaneurysm) with a high risk of serious bleeding [15].

The clear advantage of ablation is the low incidence of complications. Early complications, up to $24 \mathrm{~h}$ after the procedure, include hematomas. Hematomas can occur in subcapsular or perirenal space and any bleeding into the collecting system can cause hematuria. Skin defects including skin burns, urinomas, and transitory lumbar pain can occur. The inci-
Photo 3. Complications. Small peritumoral hematomas (arrow) on CT right after ablation (A, B). Our most serious complication - hematoma in retroperitoneum and psoatic region on contrast medium enhanced $\mathrm{CT}$ examination 1 month after the ablation (C). In all cases was conservative treatment suitable, hematomas resolved within next treatment care controls

dence of these complications varies from $7 \%$ to $17 \%$ $[6,7,16]$. In the vast majority of cases conservative treatment is sufficient. Complications that occur later and are detected in the follow-up appear in the original location of the tumor. Post-treatment care and contrast-enhanced imaging examinations are performed at intervals of 1, 6 and 12 months after the initial procedure and once each year thereafter $[6,17]$. Some elderly patients with small renal tumors are actively monitored without any surgical or interventional treatment $[18,19]$. They are often urologically asymptomatic and other diseases are much more serious and important for their life expectancy. 

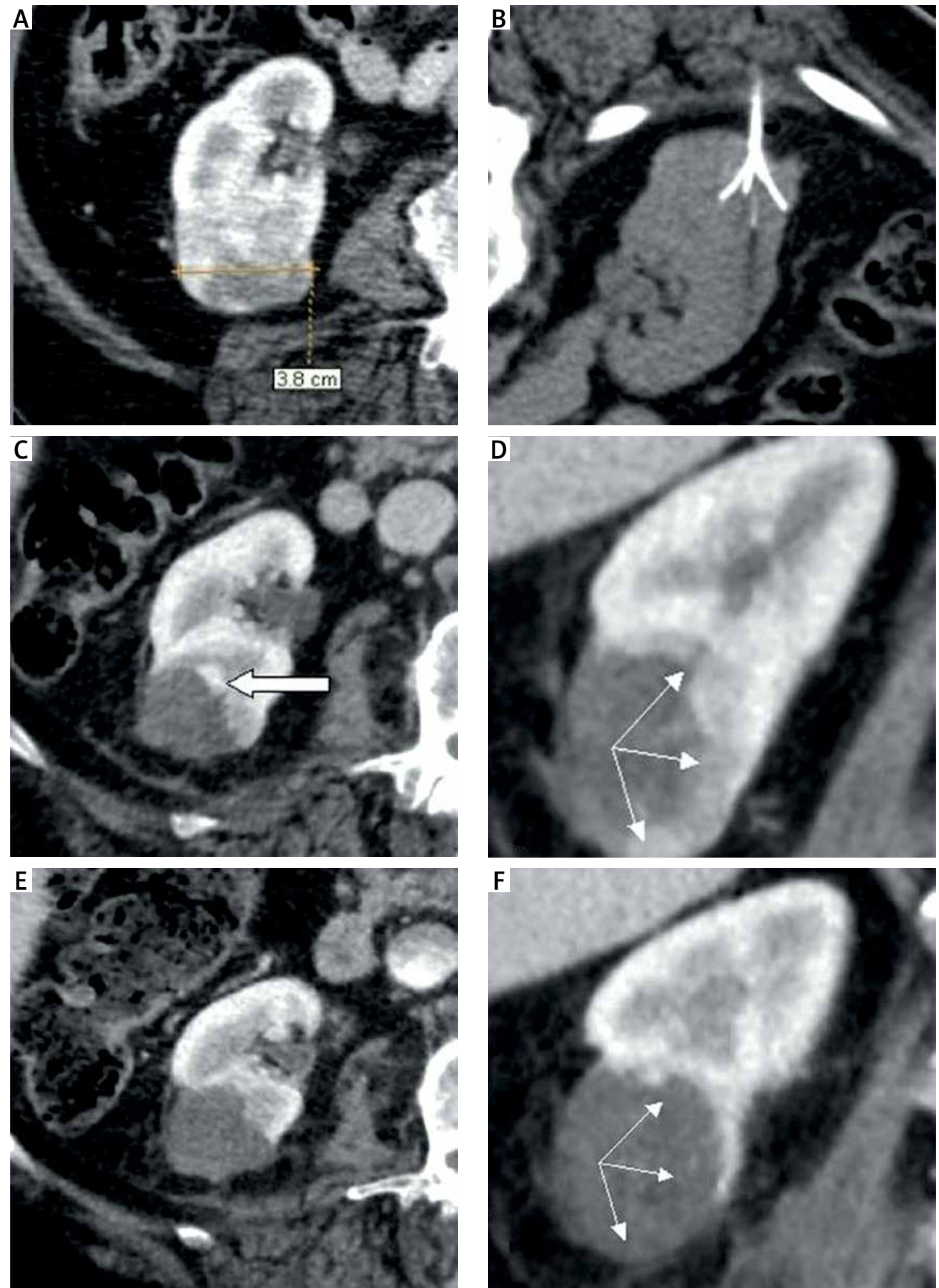

Photo 4. Nonablation. Computed tomography T examination with intravenous administration of contrast medium. Preprocedural finding of the hypervascularized tumor of length $38 \mathrm{~mm}$ (A). Radiofrequency ablation of the tumor (B). After ablation treatment care in interval 1 month verified residual tumorous tissue (arrows, C, D). Then the reablation procedure was performed and after next 1 month none tumorous process is revealed (arrows, E, F) 


\section{Conclusions}

Ablation procedures using a percutaneous approach performed with imaging guidance are very effective in the treatment of small renal tumors of the T1a group. They can be used relatively easily, can be repeated for total destruction of larger tumors, and have a low complication rate. Ablation can be a comparable alternative to surgical resection in tumors of solitary kidneys and patients with serious medical comorbidities.

\section{Acknowledgments}

The authors are grateful to Dr. Olga Cermakova for considerable help with statistical data analysis and to Jennifer Raak BA for language correction of the manuscript.

\section{Conflict of interest}

The authors declare no conflict of interest.

\section{References}

1. Jemal A, Siegel R, Ward E, et al. Cancer statistics. CA Cancer J Clin 2007; 57: 43-66.

2. American Cancer Society. Cancer Facts \& Figures 2012. American Cancer Society Inc, Atlanta 2012.

3. American Cancer Society. Cancer Facts \& Figures 2017. American Cancer Society, Atlanta 2017.

4. Dähnert W. Radiology Review Manual. $7^{\text {th }}$ ed. Lippincott Williams \& Wilkins, Philadelphia 2011; 1264

5. Sprenkle PC, Power N, Ghoneim T, et al. Comparison of open and minimally invasive partial nephrectomy for renal tumors 4-7 centimeters. Eur Urol 2012; 61: 593-9.

6. Gervais DA, MacGovern FJ, Arellano RS, et al. Radiofrequency ablation of renal cell carcinoma: part 2, Lessons learned with ablation of 100 tumors. AJR Am J Roentgenol 2005; 185: 72-80.

7. Breen DJ, Rutherford EE, Stedman B, et al. Management of renal tumors by image-guided radiofrequency ablation: experience in 105 tumors. Cardiovasc Intervent Radiol 2007; 30: 936-42.

8. Martin J, Athreya S. Meta-analysis of cryoablation versus microwave ablation for small renal masses: is there a difference in outcome? Diagn Interv Radiol 2013; 19: 501-7.

9. Narayanan G, Doshi MH. Irreversible electroporation (IRE) in renal tumors. Curr Urol Rep 2016; 17: 15.

10. Klingler C, Margreiter M, Marberger M. New ablative treatments for small renal masses: HIFU ablation. J Endourol 2013; 66: 79-89.

11. Leao RR, Richard PO, Jewett MA. The role of biopsy for small renal masses. Int I Surg 2016; 36: 513-7.

12. Ng CS, Wood CG, Silverman PM. Renal cell carcinoma: diagnosis, staging, and surveillance. AJR Am J Roentgenol 2008; 191 1220-32.
13. Ginat DT, Saad WE. Bowel displacement and protection techniques during percutaneous renal tumor thermal ablation. Tech Vasc Interv Radiol 2010; 13: 66-74.

14. Laganosky DD, Filson CP, Master VA. Surgical margins in nephron-sparing surgery for renal cell carcinoma. Curr Urol Rep 2017; 18: 8.

15. Netsch C, Bruning R, Bach T, Gross AJ. Management of renal artery pseudoaneurysm after partial nephrectomy. World J Urol 2010; 28: 519-24.

16. Gervais DA, MacGovern FJ, Arellano RS, et al. Radiofrequency ablation of renal cell carcinoma: part 1, indications, results, and role in patient management over a 6-year period and ablation of 100 tumors. AJR Am J Roentgenol 2005; 185: 64-71.

17. Ma Y, Bedir S, Cadeddu JA, Gahan JC. Long-term outcomes in healthy adults after radiofrequency ablation of T1a renal tumours. BJU Int 2014; 113: 51-5.

18. Volpe A. The role of active surveillance of small renal masses. Int J Surg 2016; 36: 518-24.

19. Jewett MA, Mattar K, Basiuk J, et al. Active surveillance of small renal masses: progression patterns of early stage kidney cancer. Eur Urol 2011; 60: 39-44.

Received: 14.08.2017, accepted: 2.10.2017. 